\title{
Habenula: Crossroad between the Basal Ganglia and the Limbic System
}

\author{
Okihide Hikosaka, ${ }^{1}$ Susan R. Sesack, ${ }^{2}$ Lucas Lecourtier, ${ }^{3}$ Paul D. Shepard ${ }^{4}$ \\ ${ }^{1}$ Laboratory of Sensorimotor Research, National Eye Institute-National Institutes of Health, Bethesda, Maryland 20892-4435, ${ }^{2}$ Departments of \\ Neuroscience and Psychiatry, University of Pittsburgh, Pittsburgh, Pennsylvania 15260, ${ }^{3}$ Laboratoire d'Imagerie et de Neurosciences Cognitives, Unité \\ Mixte de Recherche 7191, Centre National de la Recherche Scientifique, Université Louis Pasteur, 67000 Strasbourg, France, and ${ }^{4}$ Department of Psychiatry, \\ University of Maryland School of Medicine and Maryland Psychiatric Research Center, Baltimore, Maryland 21228
}

There is a growing awareness that emotion, motivation, and reward values are important determinants of our behavior. The habenula is uniquely positioned both anatomically and functionally to participate in the circuit mediating some forms of emotive decision making. In the last few years there has been a surge of interest in this structure, especially the lateral habenula (LHb). The new studies suggest that the LHb plays a pivotal role in controlling motor and cognitive behaviors by influencing the activity of dopamine and serotonin neurons. Further, dysfunctions of the LHb have also been implicated in psychiatric disorders, such as depression, schizophrenia, and druginduced psychosis.

Key words: reward; motivation; cognition; dopamine; striatum; serotonin; depression; schizophrenia

\section{Introduction}

The habenula is a pair of small nuclei located above the thalamus at its posterior end close to the midline (Fig. 1). It is regarded as part of the epithalamus, which includes the pineal body and the habenula. In many vertebrates, the habenula is divided into the medial habenula $(\mathrm{MHb})$ and the lateral habenula ( $\mathrm{LHb})$. The habenula is a phylogenetically well preserved structure that was thought to have evolved in close relation to the pineal body (Concha and Wilson, 2001; Butler and Hodos, 2005). However, recent studies using behaving animals and human subjects have instead suggested that the habenula is involved in motivational control of behavior. There have been extensive reviews on the neural connections and the functions of the habenula (Sutherland, 1982; Klemm, 2004; Lecourtier and Kelly, 2007; Geisler and Trimble, 2008). In this article we focus on the functions of the LHb, particularly in relation to its efferent connection to dopamine (DA) neurons.

\section{Connections between the $\mathrm{LHb}$, basal ganglia, and limbic system}

The LHb is comprised of multiple subnuclei that collectively form medial and lateral divisions (Andres et al., 1999). The medial division of the LHb receives afferents (Fig. 2) primarily from limbic brain regions that are directly or indirectly innervated by

Received July 23, 2008; revised Sept. 24, 2008; accepted Sept. 25, 2008.

This work was supported by the intramural research program of the National Eye Institute to $0 . H$. and by United States Public Health Service Grants MH-067937 to S.R.S., R37-MH48404 (principal investigator: B. Moghaddam) to L.L., and MH-072647 to P.D.S. (co-principal investigator: J. Gold).

Correspondence should be addressed to Okihide Hikosaka, Laboratory of Sensorimotor Research, National Eye Institute-National Institutes of Health, Building 49, Room 2A50, 49 Convent Drive, Bethesda, MD 20892-4435. E-mail: oh@|sr.nei.nih.gov.

DOI:10.1523/JNEUROSCI.3463-08.2008

Copyright $\odot 2008$ Society for Neuroscience $\quad$ 0270-6474/08/2811825-05\$15.00/0 the cerebral cortex: the lateral hypothalamic and lateral preoptic areas, basal forebrain structures including the ventral pallidum, substantia innominata, and diagonal band, and parts of the extended amygdala, including the bed nucleus of the stria terminalis (Herkenham and Nauta, 1977; Hikosaka, 2007a; Geisler and Trimble, 2008). The lateral division of the LHb is mainly innervated by the basal ganglia, in particular the globus pallidus internal segment (rodent entopeduncular nucleus), which receives cortical input by way of the striatum. Through these parallel circuits, extensive information processed by the cerebral cortex ultimately funnels to the $\mathrm{LHb}$, which serves as a convergence point for limbic and basal ganglia circuits. The efferents of the LHb (Fig. 2) primarily descend to brainstem structures but include less dense ascending projections to forebrain regions (Herkenham and Nauta, 1979; Araki et al., 1988; Hikosaka, 2007a; Geisler and Trimble, 2008). Within the brainstem, LHb efferents mainly target the nuclei containing monoamine neurons: the dopaminergic ventral tegmental area (VTA) and substantia nigra pars compacta $(\mathrm{SNc})$, serotonergic dorsal and median raphe, and cholinergic laterodorsal tegmentum. Some DA neurons in the VTA project back to the LHb (Gruber et al., 2007). Hence, the LHb forms a node of connection between the cortex and brainstem monoamine neurons that operates in parallel to the medial forebrain bundle. LHb neurons are heterogeneous in their neurochemical expression patterns, although the majority appear to have a glutamatergic phenotype (Geisler and Trimble, 2008). In their physiological regulation of brainstem DA and serotonin neurons, LHb cells exert a relatively short latency and potent inhibitory influence (Wang and Aghajanian, 1977; Christoph et al., 1986; Park, 1987; Shepard et al., 2006; Ji and Shepard, 2007; Lecourtier and Kelly, 2007; Matsumoto and Hikosaka, 2007).

The results of physiological experiments indicate a probable disynaptic circuit involving LHb projections to intermediate, 
short-range GABA cells that ultimately inhibit monoamine cell firing. Hence, it can be hypothesized that $\mathrm{LHb}$ projections to the VTA synapse onto GABA cells and not DA neurons, thus providing an indirect inhibitory action through local GABA connections. However, preliminary ultrastructural analyses have so far failed to support this hypothesis (Bell et al., 2007). Specifically, LHb axons with excitatory morphology have been found to synapse directly onto DA neurons, and conversely, excitatory-type synapses onto GABA cells have not yet been observed (Bell et al., 2007). Additional investigation is necessary before a disynaptic inhibitory circuit involving VTA GABA neurons can be ruled out. Nevertheless, it must be considered that the dominant inhibition of DA neurons induced by the $\mathrm{LHb}$ may involve GABA cells that are extrinsic to the VTA. Similar circuit questions remain to be addressed in the raphe nuclei and other brainstem targets of the LHb.

\section{Motor functions of the $\mathrm{LHb}$}

The inhibitory effects of the LHb on DA neurons are crucial for reward-based motor control. DA neurons carry signals that are essential for initiation and learning of body movements. First, they represent reward prediction error (a difference between the actual reward value and the expected reward value), which is thought to act as a teaching signal for motor behavior that would maximize the gain of reward (Schultz, 1998). Second, they encode novel environmental events that would cause immediate changes in motor behavior (Redgrave and Gurney, 2006). DA neurons in the SNc mainly project to the dorsal and ventral striatum and are thought to modulate the effectiveness of the inputs to the striatum, especially those from the cerebral cortex (Reynolds and Wickens, 2002). Indeed, the sensorimotor activity of many neurons in the striatum and other basal ganglia areas is modulated by the expected reward value of the impending action (for review, see Hikosaka et al., 2006). Several lines of evidence suggest that these reward modulations are caused, at least in part, by the DA inputs to the striatum (Nakamura and Hikosaka, 2006). Thus, the basal ganglia contribute to the selection of body movements (or actions) based on their reward outcomes.

A recent study suggested that the LHb plays an instructive role in reward-based action selection (Matsumoto and Hikosaka, 2007). Monkeys were trained to make a saccade to one of two targets, but the amount of reward after the saccade was determined by the position of the target. LHb neurons were inhibited by the target indicating a large reward and excited by the target Figure 1.
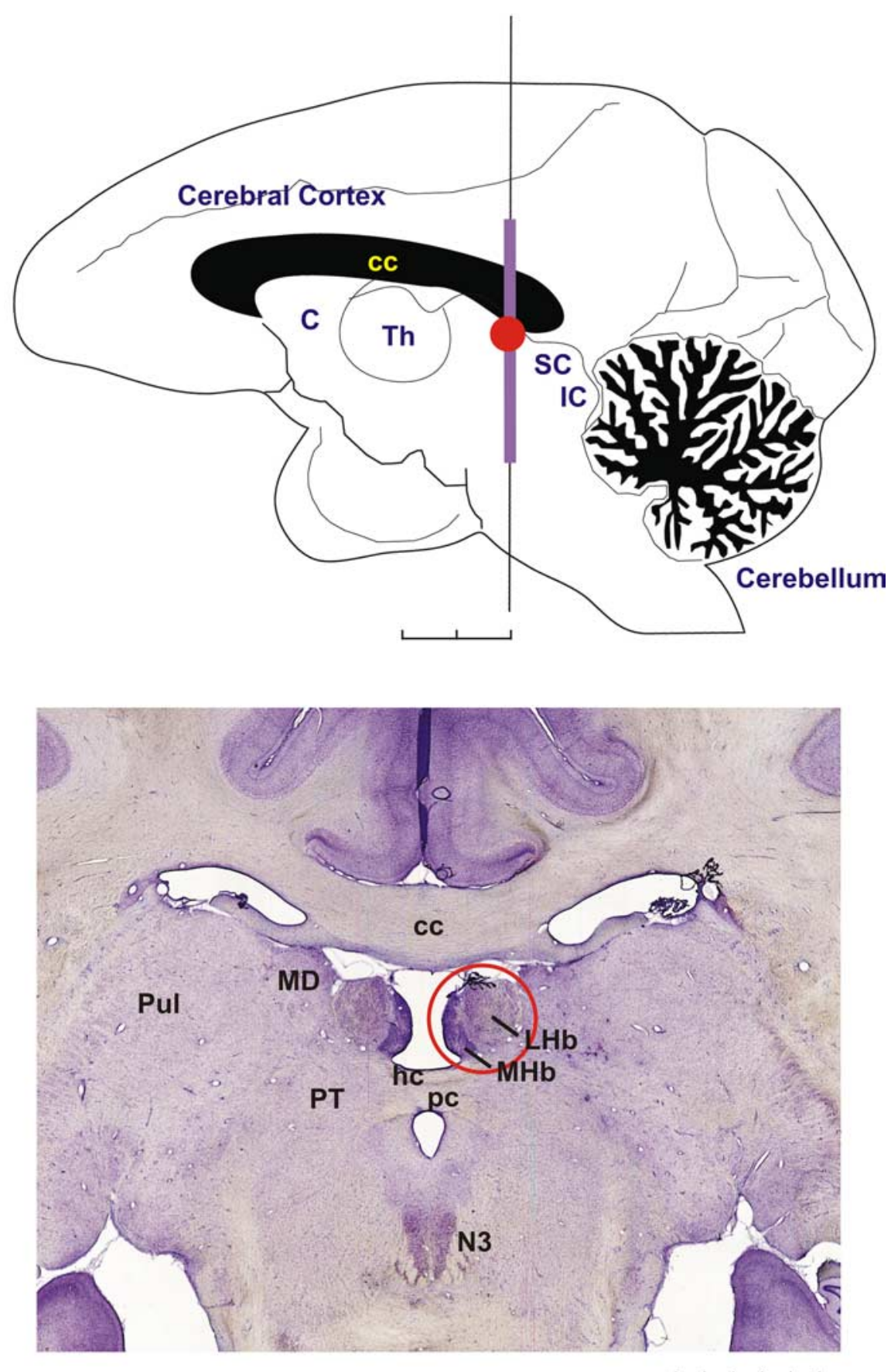

Figure 1. The habenula in the rhesus monkey. Top, The monkey's brain viewed from the mesial side. The location of the habenula is indicated by a red circle. C, Caudate nucleus; Th, thalamus; SC, superior colliculus; IC, inferior colliculus. Scale bar, 5 $\mathrm{mm} \times 2$. Bottom, A coronal histological section showing the habenula (red circle). The medially located dark region corresponds to the $\mathrm{MHb}$, whereas the lateral part corresponds to the LHb. The vertical extent of this section corresponds to a violet line in the top figure. MD, Mediodorsal nucleus of the thalamus; Pul, pulvinar; PT, pretectum; N3, oculomotor nucleus; hc, habenular commissure; pc, posterior commissure. Scale bar, $1 \mathrm{~mm} \times 5$. This figure was reprinted with permission from Hikosaka (2007a), his

indicating a small reward. They responded to the reward only when it was not predicted: inhibited by unexpectedly large rewards and excited by unexpectedly small rewards. These response patterns were exactly opposite to those of DA neurons. The excitation of LHb neurons in response to a small reward-predicting target started before the inhibition of DA neurons in the same condition. Further, weak electrical stimulation in the LHb induced clear inhibitions in DA neurons, consistent with other 


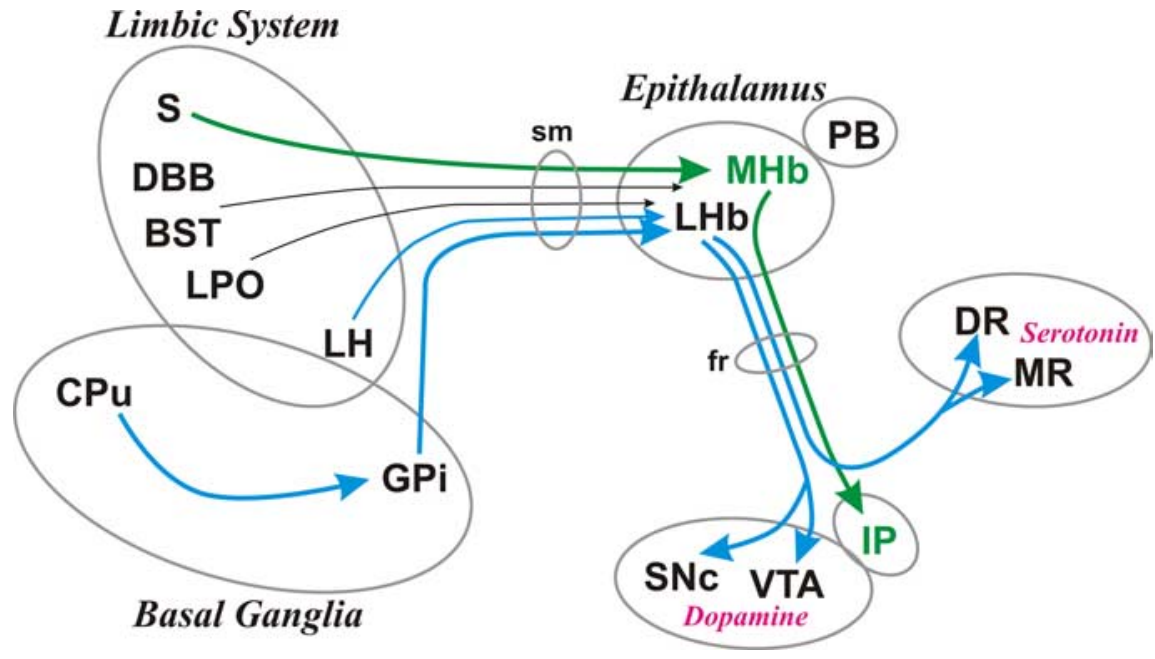

Figure 2. Afferent and efferent connections of the habenula. The $\mathrm{MHb}$, $\mathrm{LHb}$, and pineal body (PB) are collectively called the epithalamus. The $\mathrm{MHb}$ receives inputs mainly from the supracommissural septum $(S)$ and sends outputs to the interpeduncular nucleus (IP). The LHb receives inputs mainly from the basal ganglia and limbic regions and sends outputs to the brain structures containing DA neurons and serotonin neurons. Afferent and efferent connections of the habenula are conveyed by the stria medullaris (sm) and fasciculus retroflexus (fr), respectively. Green and blue lines indicate the axonal connections associated with the MHb and LHb, respectively; black lines are associated with both. The thickness of the line implies the strength of the connection. Many other connections are not shown, including reverse connections (e.g., from DR/VTA to LHb). DR, Dorsal raphe; MR, medial raphe; S, septum; DBB, nucleus of diagonal band of Broca; BST, bed nucleus of stria terminalis; LPO, lateral preoptic area; $\mathrm{LH}$, lateral hypothalamus; $\mathrm{GPi}$, globus pallidus internal segment; $\mathrm{CPu}$, caudate and putamen; $\mathrm{sm}$, stria medullaris; fr, fasciculus retroflexus. This figure was modified with permission from Hikosaka (2007a), his Figure 2.

reports (Ji and Shepard, 2007). These results suggest that the LHb suppresses less rewarding motor behaviors by inhibiting DA neurons.

\section{Cognitive functions of the $\mathrm{LHb}$}

In addition to its functions in motivational and motor control of behavior, a role for the $\mathrm{LHb}$ in cognition has recently been emphasized. In rats, studies of brain metabolic activity showed a selective activation of the LHb after the retrieval phase of an odor discrimination task for food reward (Tronel and Sara, 2002). Also, there was a decreased metabolism in the LHb during a water-maze task in aged memory-impaired rats compared with young unimpaired animals (Villarreal et al., 2002). Lesions of the habenula (including $\mathrm{LHb}$ and $\mathrm{MHb}$ ) in rats induced impairments of spatial reference memory, a model of declarative memory (Lecourtier et al., 2004), and attention (Lecourtier and Kelly, 2005). In the latter study, using the five-choice serial reaction time task (5-CSRTT), two types of deficits were observed, i.e., an immediate occurrence of an impulsive mode of behavior, represented by a large increase in premature responding, and a lateoccurring decline of accuracy. Finally, habenula lesions depress synaptic plasticity in the hippocampus-nucleus accumbens pathway (Lecourtier et al., 2006), involved in many cognitive processes.

Several lines of evidence point to alterations of subcortical DA transmissions as being one of the effectors of habenula lesioninduced cognitive deficits. In fact, the LHb tonically inhibits DA transmission, as evidenced by a marked and sustained increase in DA release in the striatum and nucleus accumbens (Lecourtier et al., 2008). The latter findings might explain the occurrence of impulsivity in the 5-CSRTT after habenula lesions, as well as the postlesion depressed plasticity of the hippocampus-nucleus accumbens pathway, given that similar plasticity changes occur with increased DA tone (Beurrier and Malenka, 2002; Hunt et al., 2005).
On the other hand, impaired spatial reference memory induced by habenula lesions might involve other neurotransmitter systems such as serotonin and acetylcholine. Indeed, the $\mathrm{LHb}$ participates in the regulation of the cholinergic medial septum (MS)-hippocampus pathway, which is involved in spatial reference memory. Also, the LHb modulates the activity of raphe serotonin neurons (Wang and Aghajanian, 1977) and serotonin transmission (Reisine et al., 1982), whereas serotonin regulates the neuronal activity of the MS and consequently of the MS-hippocampus pathway (Cassel and Jeltsch, 1995; Koenig et al., 2008). Finally, the fact that habenula lesions induced a hypersensitivity to stress could also account for some cognitive deficits (Thornton and Davies, 1991; Heldt and Ressler, 2006).

\section{Clinical implications}

Given the reciprocal connections between the $\mathrm{LHb}, \mathrm{DA}$ and serotonin neurons, it is not surprising that these pathways have been linked to neuropsychiatric conditions in which monoamine-containing neurons have been implicated. Early studies showing that serotonin neurons in the dorsal raphe are inhibited by habenular stimulation (Wang and Aghajanian, 1977; Reisine et al., 1982; Park, 1987) led to the suggestion that increased activation of the LHb may contribute to major depressive disorders (for review, see Shumake and Gonzalez-Lima, 2003). In support of this notion, patients experiencing transient depressive episodes elicited by a reduction in dietary tryptophan show increased regional cerebral blood flow in the habenula that correlates directly with ratings of depressed mood and inversely with plasma tryptophan levels (Morris et al., 1999). Enhanced LHb metabolism and reduced brain serotonin levels have been observed in several animal models of depressed behavior and can be attenuated or blocked by antidepressant drugs (CaldecottHazard et al., 1988) or lesions of the LHb (Yang et al., 2008). Together, these data suggest that habenular efferents play a prominent role in reducing brain serotonin activity associated with some forms of depressive mood and behavior and have led to the proposition that functional inactivation of the LHb by deep brain stimulation may have utility in the treatment of major depressive illness in humans (Sartorius and Henn, 2007; Hauptman et al., 2008).

Habenular efferents also exert a powerful inhibitory influence over DA-containing neurons in the SN and VTA. Lesions of the $\mathrm{LHb}$ increase DA turnover in terminal fields (Lisoprawski et al., 1980; Nishikawa et al., 1986; Lecourtier et al., 2008), whereas local stimulation transiently inhibits the spontaneous firing of SN and VTA DA neurons (Christoph et al., 1986; Ji and Shepard, 2007). A functional magnetic resonance imaging (fMRI) study conducted in healthy human volunteers has provided evidence that the habenula participates in a circuit encoding the neural representation of negative outcomes (Ullsperger and von Cramon, 2003). Subsequent studies showing that neural activity in the LHb is transiently increased in primates experiencing the loss of an anticipated reward, and that these changes are both 
antecedent and opposite to those occurring in DA neurons, provide compelling support for the role of LHb in the generation of a teaching signal used to modify action selection (Matsumoto and Hikosaka, 2007). Many patients with DArelated disorders, including Parkinson's disease and schizophrenia, do not make optimal use of negative feedback to guide action selection and problem solving (Frank et al., 2004; Cools et al., 2006; Prentice et al., 2008), which could be attributable to disruption in the habenulomesencephalic reward circuit. Preliminary fMRI results indicate that in contrast to healthy volunteers, schizophrenics do not engage habenular activity in response to informative error (Shepard et al., 2006; Holcomb et al., 2008). These differences may partially account for the maladaptive behaviors (e.g., perseveration) exhibited by patients making inconsistent use of negative feedback.

\section{Conclusions}

Recent studies reviewed above have suggested that the LHb controls cognitive and motor behaviors based on the motivational values associated with the behaviors. Such functions are, at least partly, mediated by the inhibitory effects of the LHb on midbrain DA neurons. A hypothetical scheme in Figure 3 shows how the LHb might control motor and cognitive behaviors through its influences on DA neurons.

A robust effect occurs when LHb neurons are excited by a sensory event predicting a nonrewarding (or more accurately, less rewarding than predicted) or punishing outcome (Matsumoto and Hikosaka, 2007). This causes a transient inhibition of DA neurons (Ji and Shepard, 2007), and a reduction of DA levels, especially in the dorsal and ventral striatum. Although its functional significance is still under debate (Bayer and Glimcher, 2005), the reduction in DA transmission may then lead to suppression of motor and cognitive actions, presumably through $\mathrm{D}_{2}$ receptor-mediated LTD mechanisms (Lecourtier et al., 2006; Nakamura and Hikosaka, 2006; Hikosaka, 2007b; Kreitzer and Malenka, 2007). This mechanism thus could underlie a principle of behavior: if the outcome of an action is undesirable, avoid the action.

On the other hand, LHb neurons are inhibited by a sensory event predicting a rewarding (or more accurately, more rewarding than predicted) or nonpunishing outcome (Matsumoto and Hikosaka, 2007). This causes a disinhibition of DA neurons and a transient increase in DA concentration in the striatum (Lecourtier et al., 2008). This may lead to facilitation of motor and cognitive actions, presumably through $\mathrm{D}_{1}$-mediated LTP mechanisms (Reynolds and Wickens, 2002). This mechanism might underlie a second principle of behavior: if the outcome of an action is desirable, repeat the action.

If the LHb is damaged or does not work normally, these two functions would be deranged. Even if the outcome of an action is undesirable, it would be difficult to avoid the action. This may be expressed as impulsivity, attention deficits (Lecourtier and Kelly, 2005), stereotyped motor behaviors (Carvey et al., 1987), and increased bingeing behavior (Ellison, 2002). Or, even if the outcome of an action is desirable, it would be difficult to repeat the action. It may be expressed as deficits in motor or cognitive learning (Tronel and Sara, 2002; Lecourtier et al., 2004). These hypo- thetical deficits might have common grounds with some psychiatric symptoms (Sandyk, 1991; Shepard et al., 2006).

The LHb also exerts inhibitory control over serotonin neurons and dysfunctions of this mechanism may underlie mood disorders such as depression, as discussed above. However, it is unclear whether different kinds of information are processed between the LHb-DA pathway and the LHb-serotonin pathway. A hint on this question has been provided by a recent study showing that neurons in the monkey dorsal raphe, presumably including serotonin neurons, encode reward values, but in a manner different from DA neurons (Nakamura et al., 2008).

To summarize, the recent surge in interest in the LHb has opened up a new perspective for understanding the subcortical mechanisms of cognitive and emotive behaviors. Upcoming experimental results may be instrumental for determining how this structure might be targeted in the design of novel treatments for mental and neurological disorders.

\section{References}

Andres KH, von Düring M, Veh RW (1999) Subnuclear organization of the rat habenular complexes. J Comp Neurol 407:130-150.

Araki M, McGeer PL, Kimura H (1988) The efferent projections of the rat lateral habenular nucleus revealed by the PHA-L anterograde tracing method. Brain Res 441:319-330.

Bell RL, Omelchenko N, Sesack SR (2007) Lateral habenula projections to the ventral tegmental area in the rat synapse onto dopamine and GABA neurons. Soc Neurosci Abstr 33:780.9.

Bayer HM, Glimcher PW (2005) Midbrain dopamine neurons encode a quantitative reward prediction error signal. Neuron 47:129-141.

Beurrier C, Malenka RC (2002) Enhanced inhibition of synaptic transmission by dopamine in the nucleus accumbens during behavioral sensitization to cocaine. J Neurosci 22:5817-5822.

Butler AB, Hodos W (2005) Comparative vertebrate neuroanatomy: evolution and adaptation. Hoboken, NJ: Wiley.

Caldecott-Hazard S, Mazziotta J, Phelps M (1988) Cerebral correlates of depressed behavior in rats, visualized using ${ }^{14} \mathrm{C}$-2-deoxyglucose autoradiography. J Neurosci 8:1951-1961.

Carvey PM, Kao LC, Klawans HL (1987) The effect of bilateral kainic acidinduced lateral habenula lesions on dopamine-mediated behaviors. Brain Res 409:193-196.

Cassel JC, Jeltsch H (1995) Serotonergic modulation of cholinergic function in the central nervous system: cognitive implications. Neuroscience 69:1-41.

Christoph GR, Leonzio RJ, Wilcox KS (1986) Stimulation of the lateral habenula inhibits dopamine-containing neurons in the substantia nigra and ventral tegmental area of the rat. J Neurosci 6:613-619.

Concha ML, Wilson SW (2001) Asymmetry in the epithalamus of vertebrates. J Anat 199:63-84.

Cools R, Altamirano L, D’Esposito M (2006) Reversal learning in Parkinson's disease depends on medication status and outcome valence. Neuropsychologia 44:1663-1673. 
Ellison G (2002) Neural degeneration following chronic stimulant abuse reveals a weak link in brain, fasciculus retroflexus, implying the loss of forebrain control circuitry. Eur Neuropsychopharmacol 12:287-297.

Frank MJ, Seeberger LC, O’Reilly RC (2004) By carrot or by stick: cognitive reinforcement learning in parkinsonism. Science 306:1940-1943.

Geisler S, Trimble M (2008) The lateral habenula: no longer neglected. CNS Spectrums 13:484-489.

Gruber C, Kahl A, Lebenheim L, Kowski A, Dittgen A, Veh RW (2007) Dopaminergic projections from the VTA substantially contribute to the mesohabenular pathway in the rat. Neurosci Lett 427:165-170.

Hauptman JS, DeSalles AA, Espinoza R, Sedrak M, Ishida W (2008) Potential surgical targets for deep brain stimulation in treatment-resistant depression. Neurosurg Focus 25:E3.

Heldt SA, Ressler KJ (2006) Lesions of the habenula produce stress- and dopamine-dependent alterations in prepulse inhibition and locomotion. Brain Res 1073-1074:229-239.

Herkenham M, Nauta WJ (1977) Afferent connections of the habenular nuclei in the rat. A horseradish peroxidase study, with a note on the fiber-of-passage problem. J Comp Neurol 173:123-146.

Herkenham M, Nauta WJ (1979) Efferent connections of the habenular nuclei in the rat. J Comp Neurol 187:19-47.

Hikosaka O (2007a) Habenula. Scholarpedia 2:2703.

Hikosaka O (2007b) Basal ganglia mechanisms of reward-oriented eye movement. Ann N Y Acad Sci 1104:229-249.

Hikosaka O, Nakamura K, Nakahara H (2006) Basal ganglia orient eyes to reward. J Neurophysiol 95:567-584.

Holcomb HH, Rowland LM, Griego JA (2008) When given feedback schizophrenic learners adapt and improve in accuracy using prefrontal and striatal resources. Soc Neurosci Abstr 34:313.3.

Hunt MJ, Kessal K, Garcia R (2005) Ketamine induces dopaminedependent depression of evoked hippocampal activity in the nucleus accumbens in freely moving rats. J Neurosci 25:524-531.

Ji H, Shepard PD (2007) Lateral habenula stimulation inhibits rat midbrain dopamine neurons through a $\mathrm{GABA}_{\mathrm{A}}$ receptor-mediated mechanism. J Neurosci 27:6923-6930.

Klemm WR (2004) Habenular and interpeduncularis nuclei: shared components in multiple-function networks. Med Sci Monit 10:RA261-RA273.

Koenig J, Cosquer B, Cassel JC (2008) Activation of septal 5-HT1A receptors alters spatial memory encoding, interferes with consolidation, but does not affect retrieval in rats subjected to a water-maze task. Hippocampus 18:99-118.

Kreitzer AC, Malenka RC (2007) Endocannabinoid-mediated rescue of striatal LTD and motor deficits in Parkinson's disease models. Nature 445:643-647.

Lecourtier L, Kelly PH (2005) Bilateral lesions of the habenula induce attentional disturbances in rats. Neuropsychopharmacology 30:484-496.

Lecourtier L, Kelly PH (2007) A conductor hidden in the orchestra? Role of the habenular complex in monoamine transmission and cognition. Neurosci Biobehav Rev 31:658-672.

Lecourtier L, Neijt HC, Kelly PH (2004) Habenula lesions cause impaired cognitive performance in rats: implications for schizophrenia. Eur J Neurosci 19:2551-2560.

Lecourtier L, Deschaux O, Arnaud C, Chessel A, Kelly PH, Garcia R (2006) Habenula lesions alter synaptic plasticity within the fimbria-accumbens pathway in the rat. Neuroscience 141:1025-1032.

Lecourtier L, Defrancesco A, Moghaddam B (2008) Differential tonic influence of lateral habenula on prefrontal cortex and nucleus accumbens dopamine release. Eur J Neurosci 27:1755-1762.

Lisoprawski A, Herve D, Blanc G, Glowinski J, Tassin JP (1980) Selective activation of the mesocortico-frontal dopaminergic neurons induced by lesion of the habenula in the rat. Brain Res 183:229-234.

Matsumoto M, Hikosaka O (2007) Lateral habenula as a source of negative reward signals in dopamine neurons. Nature 447:1111-1115.

Morris JS, Smith KA, Cowen PJ, Friston KJ, Dolan RJ (1999) Covariation of activity in habenula and dorsal raphe nuclei following tryptophan depletion. Neuroimage 10:163-172.

Nakamura K, Hikosaka O (2006) Role of dopamine in the primate caudate nucleus in reward modulation of saccades. J Neurosci 26:5360-5369.

Nakamura K, Matsumoto M, Hikosaka O (2008) Reward-dependent modulation of neuronal activity in the primate dorsal raphe nucleus. J Neurosci 28:5331-5343.

Nishikawa T, Fage D, Scatton B (1986) Evidence for, and nature of, the tonic inhibitory influence of habenulointerpeduncular pathways upon cerebral dopaminergic transmission in the rat. Brain Res 373:324-336.

Park MR (1987) Monosynaptic inhibitory postsynaptic potentials from lateral habenula recorded in dorsal raphe neurons. Brain Res Bull 19:581-586.

Prentice KJ, Gold JM, Buchanan RW (2008) The Wisconsin Card Sorting impairment in schizophrenia is evident in the first four trials. Schizophr Res. Advance online publication. Retrieved October 22, 2008. doi:10.1016/j.schres.2007.07.015.

Redgrave P, Gurney K (2006) The short-latency dopamine signal: a role in discovering novel actions? Nat Rev Neurosci 7:967-975.

Reisine TD, Soubrié P, Artaud F, Glowinski J (1982) Involvement of lateral habenula-dorsal raphe neurons in the differential regulation of striatal and nigral serotonergic transmission cats. J Neurosci 2:1062-1071.

Reynolds JN, Wickens JR (2002) Dopamine-dependent plasticity of corticostriatal synapses. Neural Netw 15:507-521.

Sandyk R (1991) Relevance of the habenular complex to neuropsychiatry: a review and hypothesis. Int J Neurosci 61:189-219.

Sartorius A, Henn FA (2007) Deep brain stimulation of the lateral habenula in treatment resistant major depression. Med Hypotheses 69:1305-1308.

Schultz W (1998) Predictive reward signal of dopamine neurons. J Neurophysiol 80:1-27.

Shepard PD, Holcomb HH, Gold JM (2006) Schizophrenia in translation: the presence of absence: habenular regulation of dopamine neurons and the encoding of negative outcomes. Schizophr Bull 32:417-421.

Shumake J, Gonzalez-Lima F (2003) Brain systems underlying susceptibility to helplessness and depression. Behav Cogn Neurosci Rev 2:198-221.

Sutherland RJ (1982) The dorsal diencephalic conduction system: a review of the anatomy and functions of the habenular complex. Neurosci Biobehav Rev 6:1-13.

Thornton EW, Davies C (1991) A water-maze discrimination learning deficit in the rat following lesion of the habenula. Physiol Behav 49:819-822.

Tronel S, Sara SJ (2002) Mapping of olfactory memory circuits: regionspecific c-fos activation after odor-reward associative learning or after its retrieval. Learn Mem 9:105-111.

Ullsperger M, von Cramon DY (2003) Error monitoring using external feedback: specific roles of the habenular complex, the reward system, and the cingulate motor area revealed by functional magnetic resonance imaging. J Neurosci 23:4308-4314.

Villarreal JS, Gonzalez-Lima F, Berndt J, Barea-Rodriguez EJ (2002) Water maze training in aged rats: effects on brain metabolic capacity and behavior. Brain Res 939:43-51.

Wang RY, Aghajanian GK (1977) Physiological evidence for habenula as major link between forebrain and midbrain raphe. Science 197:89-91.

Yang LM, Hu B, Xia YH, Zhang BL, Zhao H (2008) Lateral habenula lesions improve the behavioral response in depressed rats via increasing the serotonin level in dorsal raphe nucleus. Behav Brain Res 188:84-90. 